\section{Central precocious puberty and granulosa cell ovarian tumor in an 8-year old female}

\author{
Valeria Calcaterra, 1 Ghassan Nakib, 2 \\ Gloria Pelizzo,2 Barbara Rundo,1 \\ Gaetana Anna Rispoli,3 Stella Boghen,1 \\ Federico Bonetti,4 Barbara Del Monte, 5 \\ Chiara Gertosio, ${ }^{1}$ Daniela Larizza ${ }^{1}$ \\ 1Department of the Mother and Child \\ Health, Pediatric Unit, IRCCS Policlinico \\ San Matteo Foundation and Department \\ of Internal Medicine, University of Pavia; \\ 2Department of the Mother and Child \\ Health, Pediatric Surgery Unit, IRCCS \\ Policlinico San Matteo Foundation and \\ University of Pavia; ${ }^{3}$ Department of \\ Neuroradiology, IRCCS Policlinico San \\ Matteo Foundation Pavia; 4Department \\ of the Mother and Child Health, \\ Haematologic/Oncologic Unit, IRCCS \\ Policlinico San Matteo Foundation, Pavia; \\ 5 Anatomic Pathology Section, \\ Department of Human Pathology, \\ University of Pavia, Foundation IRCCS \\ Policlinico San Matteo, Pavia, Italy
}

\section{Abstract}

Ovarian tumors associated with hormonal changes of the peripheral iso-sexual precocious puberty are of common presentation. We describe here a rare case of juvenile granulosa cell tumor in a female with central precocious puberty (CPP). An 8-year old girl with CPP presented with vaginal bleeding four months after the diagnosis and before starting treatment with gonadotropin-releasing hormone (GnRH)analogs. Suppression of basal follicle-stimulating hormone (FSH) level, elevation of serum estradiol, progesterone and Cancer Antigen-125 were documented. Abdominal ultrasound examination (US) and magnetic resonance imaging showed a pelvic mass affecting the left ovary. A left salpingo-oophorectomy was performed and the mass was totally resected. Juvenile granulosa cell ovarian tumor was diagnosed. One month post surgery, estradiol and progesterone decreased to values of the first evaluation and FSH increased; Cancer Antigen-125 resulted normal while ultrasound pelvic examination showed absence of pelvic masses. In our patient, the tumor had grown very quickly since hormonal data demonstrated a CPP without any evidence of ovarian mass on US only four months before diagnosis. The overstimulation of the FSH or aberrant activation of FSH receptors may have contributed to the development of the mass.

\section{Introduction}

Ovarian tumors are rare in children and adolescents. Malignant ovarian neoplasms comprise approximately $1 \%$ of all childhood cancers and represent the most common gynecological tumors. Eighty percent are germ cell tumors and only $5-8 \%$ of cases are of sex cord stromal origin. Granulosa cell tumors (GCTs) are a group of estrogen producing sex cord stromal tumors that account for approximately $2 \%$ of all ovarian tumors. ${ }^{1-3}$ On the basis of histological characteristics and age of onset, these tumors are divided into adult type (95\% of cases) and juvenile type (5\% of cases). Precocious pseudo-puberty, vaginal bleeding and irregular menstruation are a common presentation of these tumors associated with hormonal changes. ${ }^{1,2}$ We describe here a rare case of juvenile granulosa cell tumor (JGCT) in a girl with central precocious puberty.

\section{Case Report}

A Caucasian female with no significant past medical history presented with breast enlargement (Tanner stage B2) and pubic hair (PH2) at the age of 7 years and 9 months. At her first examination (8 years and 5 months), anthropometric evaluation revealed height $126.4 \mathrm{~cm}$ (50th percentile), weight $32 \mathrm{~kg}$ (97th percentile), and body mass index $20.3 \mathrm{~kg} / \mathrm{m}^{2}$ (75$90^{\text {th }}$ percentile). She had no headache or visual complaints and did not demonstrate any neurological signs; physical examination was unremarkable.

Endocrine evaluation revealed elevation of GnRH-stimulated luteinizing hormone (LH) and FSH peak (Table 1); other hormonal levels, related to adrenal and thyroid function, were normal. Bone age advancement (SDS-BA 2.1), without growth spurt, was found. Tumor markers were negative.

An ultrasound examination (US) showed a pubertal uterus; the left and right ovaries were normal (Figure 1A).

Based on history and pubertal hormonal profile the diagnosis of central precocious puberty was formulated. After four months, during completion of screening tests and magnetic resonance imaging (MRI) of the hypothalamic-pituitary region and before starting treatment with $\mathrm{GnRH}$ analogs, the patient presented a vaginal bleeding without other symptoms or progression of pubertal development. Physical examination evidenced a palpable fullness in the pelvic region associated with increase in abdominal girth. Suppression of basal FSH level, elevation of serum estradiol, progesterone and CA125 were observed (Table 1). Pelvic ultrasound demonstrated the presence of a
Correspondence: Daniela Larizza, Pediatric Unit, IRCCS Policlinico S. Matteo Foundation, piazzale Golgi 2, Pavia, Italy. Tel. +39.0382 .502885$

E-mail: d.larizza@smatteo.pv.it

Key words: central precocious puberty, juvenile granulosa cell, ovarian tumor, children.

Contributions: VC prepared draft and wrote manuscript; GN gave surgical support and prepared draft; GP gave surgical support; BR, SB and CG gave clinical support; GAR and BdM gave diagnostic support; FB gave oncological support; DL gave clinical and endocrinological support, and wrote manuscript.

Conflict of interests: the authors declare no potential conflict of interests.

Received for publication: 9 June 2013.

Revision received: 17 June 2013.

Accepted for publication: 8 July 2013 .

This work is licensed under a Creative Commons Attribution NonCommercial 3.0 License (CC BYNC 3.0).

(C) Copyright V. Calcaterra et al., 2013

Licensee PAGEPress, Italy

Pediatric Reports 2013; 5:e13

doi:10.4081/pr.2013.e13

huge lesion $(13 \times 8 \mathrm{~cm})$ affecting the left ovary (Figure 1B). An MRI of the abdomen confirmed the US findings of a pelvic capsulated mixed mass $(13 \times 13.7 \times 6 \mathrm{~cm})$ arising from the left ovary (Figure 1C). The girl underwent laparotomy and a left salpingo-oophorectomy, and a complete mass resection was performed. On gross examination, the tumor originated from the left ovary that was substituted by a gray mass with solid and cystic components and hemorrhagic areas (Figure 1D). The microscopic appearance was characterized by a proliferation of the sex-stromal cells with high mitotic activity and low-grade cell atypia. Juvenile granulosa cell ovarian tumor was diagnosed. Her postoperative course was unremarkable. No chemotherapy or radiation was necessary.

The girl did not start suppressive therapy with GnRH analogs in order to permit a follow up without interfering agents. At one month post surgery, estradiol and progesterone decreased to values of the first evaluation and FSH increased; CA-125 resulted normal while pelvic ultrasound showed an absence of pelvic masses. Seven months after surgery there were no signs of re-occurrence of tumor. MRI of the hypothalamic pituitary region revealed the presence of a pineal cyst.

\section{Discussion}

Precocious puberty (PP) in girls is defined 
as the development of secondary sexual characteristics before the age of eight years. Two types of PP are recognized: central precocious puberty (CPP) and peripheral precocious puberty (PPP). CPP is caused by early activation of the hypothalamus-pituitary-gonadal axis. It is usually idiopathic but secondary causes include tumors, infections, congenital defects, and radiation or injury to the brain. PPP does not involve the HPG axis. It is caused by release of estrogen or testosterone from abnormal organs and causes include gonadal tumor. 4

Ovarian tumors are uncommon lesions in children. Their approximate incidence is estimated at approximately 2.6 cases annually per 100,000 girls, and malignant ovarian tumors represent approximately $1 \%$ of all childhood cancers. ${ }^{2}$ The incidence of GCTs, which is a subset of sex cord stromal tumors, varies from 0.4 to 1.7 cases per 100,000 women. Only approximately $5 \%$ of all GCTs cases are of juvenile type and occur in pre-menarchal girls and young women.1-3

The presenting complaints in patients with ovarian masses are non-specific, making diagnosis difficult. Acute or chronic abdominal pain and abdomino-pelvic mass are the most common initial symptoms. Endocrine signs (pseudo-precocious puberty, signs of virilization, vaginal secretions, irregular uterine bleeding) can reveal some secreting ovarian tumors. ${ }^{5}$ We describe a rare case in which ovarian JGCT occurred with vaginal bleeding four months after diagnosis of central precocious puberty.

Central precocious puberty has been previously reported in some girls with ovarian GCTs and in these patients the signs of puberty developed concomitantly with the mass or some years after its removal. Gonadotropin stimulation might play a role in the development of the ovarian tumors. ${ }^{6}$ In our patient, both hormonal data and US images demonstrated a CPP without ovarian masses only four months before diagnosis. Given this, the possibility that overstimulation of the FSH may have contributed to the development of the mass cannot be ruled out; stimulation of the ovary by gonadotropin might have a direct car-

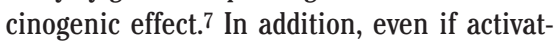
ing mutations of FSHR were not investigated, the growth of ovarian tumor in our patient might have been caused by aberrant activation of FSHR.

Age, presenting symptoms, and size of the ovarian tumor are not predictive of malignancy, but imaging features and tumor markers are useful. US is the imaging modality of choice in cases of ovarian masses in children, particularly for the initial workup; the presence of more solid components in the tumor is a definite feature that predicts malignancy. CT scan and MRI are useful in cases of undefined
Table 1. Hormonal data and serum tumor markers at diagnosis and during follow up.

\begin{tabular}{lccc} 
& $\begin{array}{c}\text { At diagnosis of central } \\
\text { precocious puberty }\end{array}$ & $\begin{array}{c}\text { At diagnosis of granulosa } \\
\text { cell tumor }\end{array}$ & $\begin{array}{c}\text { Post } \\
\text { surgery }\end{array}$ \\
Basal LH (mU/mL) & 0.2 & 0.2 & 0.1 \\
Peak LH (mU/mL) & 6.4 & n.e. & n.e. \\
\hline Basal FSH (mU/mL) & 2.3 & 0.1 & 0.6 \\
Peak FSH (mU/mL) & 13.4 & n.e. & n.e. \\
\hline $18-\beta$-estradiol (pg/mL) & 10.1 & 65 & 4.7 \\
CA125 (U/mL; n.v. 0-35) & 9.1 & 74.3 & 8.9 \\
\hline Progesterone (ng/mL) & 0.2 & 1.7 & 0.2 \\
CEA (ng/mL; n.v. 0-5) & $<0.5$ & 0.5 & $<0.5$ \\
\hline AFP $(\mathrm{U} / \mathrm{mL} ;$ n.v. 0-12) & 5.8 & 5.4 & 5.8 \\
$\beta$ BhCG (mU/mL; n.v. 0-5) & $<2$ & $<2$ & $<2$ \\
\hline
\end{tabular}

LH, luteinizing hormone; FSH, follicle-stimulating hormone; CA125, Cancer Antigen-125; CEA, carcinoembryonic antigen; AFP, alpha-fetoprotein; hCG, beta human chorionic gonadotropin; n.v., normal values; n.e., not evaluated.

\section{Table 2. Ovarian tumor markers.}

Histology FP BhCG LDH CA-125 CEA CA 19.19 Inhibin

\section{Germinal tumors}

\begin{tabular}{lccccccc} 
Endodermal sinus tumor & + & - & + & + & - & + & - \\
\hline Teratoma (immature) & + & - & - & + & - & + & - \\
Dysgerminoma & - & + & + & + & - & + & - \\
\hline Embryonal CA & + & + & - & - & - & + & - \\
Chorio CA & - & + & - & - & - & + & - \\
\hline Epithelial cell tumors & + & + & + & + & + & + & - \\
Sex stromal cell tumors & + & - & + & + & - & - & + \\
\hline
\end{tabular}

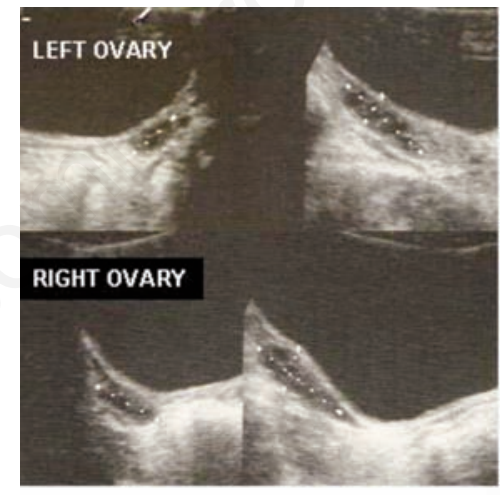

A

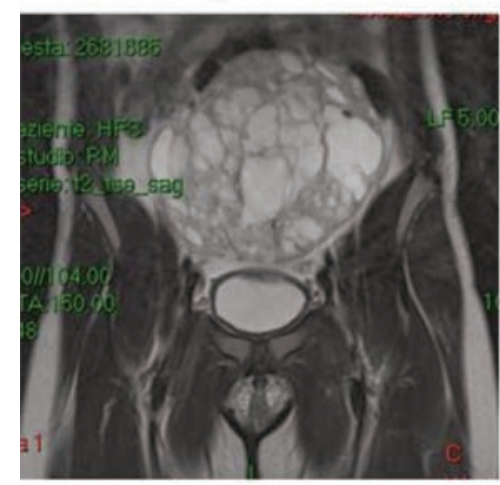

C

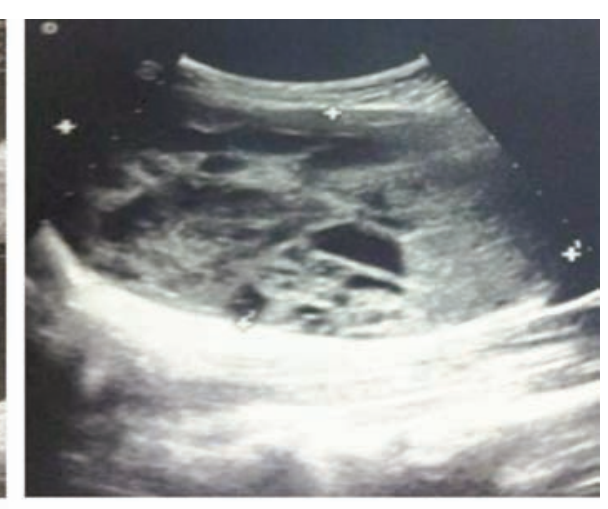

B

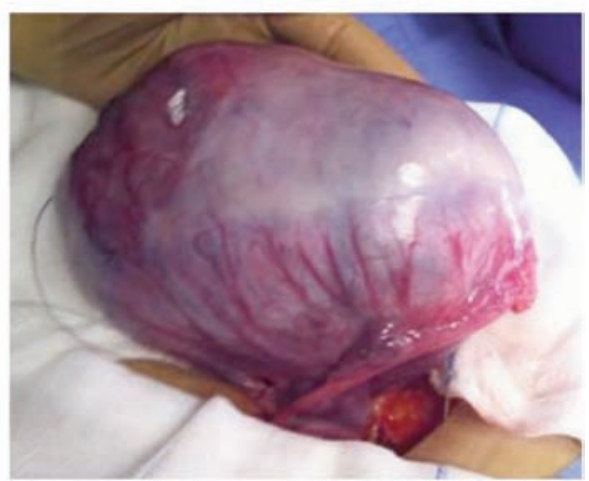

D
Figure 1. A) Ultrasound images of the ovaries at diagnosis of central precocious puberty. Ultrasound images (B), magnetic resonance imaging (C) and gross examination (D) of the ovarian mass. 
mass if malignancy is suspected and before any surgery. They give additional information about the nature of the tumor and the presence of pelvic and para-aortic lymph nodes and, therefore, increases the accuracy of the diagnosis of ovarian malignancy. 8

The evaluation of serum tumor markers, even if these are often non-specific, is very important for the differential diagnosis in pediatric ovarian masses. The surgical management and approach will be based on test results and examination findings with the aim of preserving the girl's fertility.5,9,10 Tumor markers such as AFP, $\beta$-hCG, CA 125, CEA, CA 19.9 and inhibin could indicate the presence of ovarian malignancy (Table 2).

Thus, associating the marker findings to clinical symptoms together with imaging reports, intraoperative aspect and an eventual intraoperative histopathology are all recommended to promote a confident differential diagnosis.

Ovarian malignancy at any age is treated by oophorectomy or salpingo-oophorectomy of the affected adnexa and surgical staging. 11 The prognosis of ovarian tumors in children is excellent if they are detected in an early stage. ${ }^{4}$ The risk of re-occurrence of benign tumors in the controlateral ovary is approximately $10 \%$ thus requiring regular ultrasound monitoring. Malignant tumors have a good prognosis for survival if treated properly. ${ }^{2}$

\section{Conclusions}

In conclusion, in our patient, JGCT was detected after the diagnosis of central precocious puberty. The pathogenesis of ovarian GCT is still unknown, but in this case the overstimulation of the FSH or aberrant activation of FSH receptors may have contributed to the development of the mass. The association between central precocious puberty and granulosa cell tumors might not be coincidental.

\section{References}

1. Merras-Salmio L, Vettenranta K, Möttönen M, Heikinheimo M. Ovarian granulosa cell tumors in childhood. Pediatr Hematol Oncol 2002;19:145.

2. Von Allmen D. Malignant lesions of the ovary in childhood. Semin Pediatr Surg 2005;14:100.

3. Ashnagar A, Alavi S, Nilipour Y, et al. Massive ascites as the only sign of ovarian juvenile granulosa cell tumor in an adolescent: a case report and a review of the literature. Case Rep Oncol Med 2013;2013: 386725.

4. Eugster EA, Palmert MR. Precocious puberty. J Clin Endocrinol Metab 2006; 91:0.

5. Al Jama FE, Al Ghamdi AA, Gasim T, et al. Ovarian tumors in children and adolescents: a clinical study of 52 patients in a university hospital. J Pediatr Adolesc Gynecol 2011;24:25.

6. Bas F, Pescovitz OH, Steinmetz R. No activating mutations of FSH receptor in four children with ovarian juvenile granulosa cell tumors and the association of these tumors with central precocious puberty. $\mathrm{J}$ Pediatr Adolesc Gynecol 2009;22:173.

7. Willemsen W, Kruitwagen R, Bastiaans B, et al. Ovarian stimulation and granulosacell tumour. Lancet 1993;341:98698.

8. Cartault A, Caula-Legriel S, Baunin C, et al. Ovarian masses in adolescent girls. Endocr Dev 2012;22:194.

9. Oltman SC, Garcia N, Barber R, et al. Can we preoperatively risk stratify ovarian masses for malignancy? J Pediatr Surg 2010;45:130.

10. Spinelli C, Pucci V, Buti I, et al. The role of tumor markers in the surgical approach of ovarian masses in pediatric age: a 10-year study and a literature review. Ann Surg Oncol 2012;19:1766.

11. Cass DL, Hawkins E, Brandt ML, et al. Surgery for ovarian masses in infants, children and adolescents: 102 consecutive patients treated in a 15 -year period. $\mathrm{J}$ Pediatr Surg 2001;36:69. 